\title{
DEVELOPMENT OF A MORE APPLIED VERSION OF COHERENCY CALLED 'SENSIBLE COHERENCY' FOR ASSESSMENT OF FINANCIAL RISK MEASURES
}

\author{
M. Jasemi ${ }^{1}$, A.M. Kimiagari ${ }^{2}$ and A. Memariani ${ }^{3}$ \\ ${ }^{1}$ Department of Industrial Engineering \\ Amirkabir University of Technology, Iran \\ Miladj@aut.ac.ir \\ ${ }^{2}$ Department of Industrial Engineering \\ Amirkabir University of Technology, Iran \\ kimiagar@aut.ac.ir \\ ${ }^{3}$ Department of Industrial Engineering \\ Bu-Ali Sina University, Iran
}

\begin{abstract}
Coherency is becoming a necessary feature for any risk measure, and now is an acceptable tool in risk management to assess the risk measures. For example, recent studies have strongly criticised VaR-based models for not providing a coherent risk measure. Because of such acceptance, it is important to improve the efficiency of the touchstone for evaluating risk measures in order to achieve a fairer assessment. This is just the challenge that this paper seeks to address. This goal is achieved on the one hand by doing some simplifications in axioms of coherency without losing their major financial points, and on the other hand by removing the paradox between two of the axioms. The new concept is called 'sensible coherency', and the risk measure that satisfies the four new simplified and corrected axioms will be 'sensibly coherent'. Finally, the new axioms are applied to a particular type of lower partial moments as a case study.
\end{abstract}

\section{OPSOMMING}

Koherensie word ' $n$ noodsaaklike kenmerk van enige risikomaatstaf en is nou ' $n$ aanvaarbare gereedskapstuk in die beoordeling van risikomaatstawwe. Die doel van hierdie artikel word bereik deur enersyds die aksiomas van koherensie te vereenvoudig en andersyds die paradoks tussen die aksiomas te verwyder. Die resultaat word "sinvolle koherensie" genoem. 


\section{INTRODUCTION}

In recent years a growing interest in the optimal portfolio problem, introduced by the seminal work of Markowitz [1], has become very apparent. This interest is due to two overlapping reasons. On the one side, computational advances in mathematical programming - both quantitatively and qualitatively - mean that the technology for solving financial problems with more variables or with more real assumptions, which could not be solved in Markowitz's time, is now available. In the quantitative approach there is the work of authors like Konno and Suzuki [2] or Perold [3], and in the qualitative approach there is a vast volume of literature on robust optimisation, Markov chain, multi-objective decision making, possibility and fuzzy theory or Minimax modeling of the problem. On the other side, practitioners and risk managers are confident that sometimes variance is not an appropriate measure of risk, and can be better measured by different statistical methods; and so an appropriate risk measure is necessary to address the problem more efficiently. This aspect of financial literature has been enriched by the development and introduction of new risk measures like $\mathrm{VaR}$ or $\mathrm{CVaR}$, and by defining new features for better assessment of risks. The latter direction for the enrichment of the risk literature is particularly challenging, since from a practical point of view there are many propositions about an ideal risk measure, making the embedding of all of them in mathematical or statistical relations to some extent impossible. A criterion of coherency that encompasses the four axioms of subadditivity, monotonicity, translation invariance, and positive homogeneity is one of the most accepted risk assessment tools, and is now extensively used to analyse risk measures. The risk measures that satisfy all four of the above axioms are called 'coherent'.

As discussed above, this paper is related to risk measures, and may be categorised in that group of works that focus on assessment tools of financial risks. In other words, the paper intends to develop new criteria for the assessment of financial risk measures that are more helpful than previous ones. The concept developed by this paper is neither completely new nor innovative, but a revised version of the noble work of Artzner et al. [4] in developing the concept of coherency. It is not intended to question the efficacy of coherency completely, but to highlight the fact that in some cases the concept of coherency may produce irrational results that will disappear under application of the new concept of 'sensible coherency'.

The defects of the coherency criterion can be described in two ways:

1. The criteria focus on the mathematical features of risk measures more than their practical application in finance, and the applicability of some of the axioms is sacrificed by the rigidity of their definition. In other words, some criteria of coherency are suitable for mathematical operations; but from the practical point of view they encompass very special cases in a way that seems to some extent illogical.

2. Some axioms have paradoxes with some of the others.

In this paper, by applying some simplifications to the axioms of coherency and also injecting some logical points, another set of criteria for assessment of risk measures is achieved. The new set of axioms is called 'sensible coherency', while the traditional set of axioms henceforth will be called 'absolute coherency', just to distinguish the new and old versions better. From the practical point of view, the new concept is superior to absolute coherency; but from the perspective of being appropriate for mathematical processing, the absolute version is still better.

The rest of this paper is organised as follows. Section 2 focuses on the concept 'absolute coherency'; Section 3 discusses sensible coherency; and Section 4 investigates the state of coherency (absolute, sensible, or neither) of the Lower Partial Moment (LPM) of the first order. Finally, Section 5 sets out the most important conclusions of the study. 


\section{ABSOLUTE COHERENCY}

Since the notion of risk cannot be separated from the axioms underlying the definition of rational risky behavior, there is no objective definition of risk that could be unanimously accepted - i.e. there is no perfect definition of risk, and some are relatively superior to others. This superiority is usually analysed by criteria, the most acceptable of which in the literature is the concept of 'absolute coherency'.

The theory of 'absolute coherency' was initiated by Artzner et al. ([4], [5]) and developed further by Bertsimas et al. [6], Delbaen [7], Kusuoka [8], Acerbi [9], Fritelli and Rosazza Gianin [10], Acerbi and Tasche [11], Szego [12], and Inoue [13]. For example, while Artzner et al. [5] considered only discrete probability spaces, Delbaen [7] extends their definitions to include arbitrary probability spaces. Pelessoni and Vicig [14] defined absolutely coherent measures of risk over an arbitrary set of risks - a more general case than the concept of absolute coherency for risk measures over a linear space of random numbers. Giannopoulos and Tunaru [15] have shown how filtered historical simulation (FHS) can provide absolutely coherent risk estimates; and many other works relate directly or indirectly to the concept of absolute coherency. The main point, however, is the fact that the impact of absolute coherency on the financial literature has been significant, to the extent that (for example) the Value-at-Risk (VaR) measure that has been widely used in finance and insurance for capital and risk management, has fallen somewhat out of favor in recent years due to the seminal paper of Artzner et al. [5], which showed that VaR does not in general have all four coherence properties. The same thing has happened to the risk measures based on standard deviation or semi-variance. It is to be noted that VaR is absolutely coherent for elliptically distributed risks [16], and when the assets' returns have elliptically symmetric distributions, all these risk measures are coherent.

\section{SENSIBLE COHERENCY}

This concept results from a revision of the concept of absolute coherency. The concept tries to make absolute coherency more financial and less mathematical. In other words, although sensibly coherent measures may not be capable of mathematical processing like absolutely coherent measures, they can be relied on in practice more confidently. Sensible coherency is more general than absolute coherency; the latter is just a special case of the former.

Sensible coherency consists of four axioms, although two of them - subadditivity and monotonicity - are exactly the same as absolute coherency because they are completely applied. Consequently, the distinction between the two concepts of coherency lies in the two axioms of translation invariance and positive homogeneity. The risk measure of $\rho$ is called sensibly coherent if it satisfies the four axioms of subadditivity, monotonicity, sensible translation invariance, and sensible positive homogeneity.

\subsection{Subadditivity}

This axiom states that $\rho(x+y) \leq \rho(x)+\rho(y)$. Satisfaction of this axiom ensures that the diversification principle of modern portfolio theory holds, since a subadditive measure would always generate a lower risk measure for a diversified portfolio than for a nondiversified portfolio. In terms of internal risk management, subadditivity also implies that the overall risk of a financial firm is equal to or less than the sum of the risks of individual departments of the firm.

\subsection{Monotonicity}

This axiom states that $\rho(x) \leq \rho(y)$ if $x \geq y$. That is, a monotonous risk measure never considers investment opportunities with higher returns riskier than others with lower returns. 


\subsection{Sensible translation invariance (STI)}

A risk measure is sensibly translation invariant if it satisfies the following condition:

$$
\rho(x+a)=\rho(x)-g(a) \text {; where } \quad g(a)=\left\{\begin{array}{lll}
>0 & \text { if } & a>0 \\
<0 & \text { if } & a<0
\end{array} \text { and } \frac{\partial g(a)}{\partial(a)}>0 .\right.
$$

So there is no difference between absolute and sensible coherency on the axiom of translation invariance if $g(a)=a$. Or, expressed better: absolute translation invariance (ATI) is just a special case of STI, and there is no inconsistency between them.

The impractical point with ATI is its zero one approach toward risk measures. Consider, for example, three risk measures of $\rho_{1}, \rho_{2}$ and $\rho_{3}$ where $\rho_{1}(x+a)=\rho_{1}(x)-a, \rho_{2}(x+a)=\rho_{2}(x)-a \pm \varepsilon$ and $\rho_{3}(x+a)=f(a) \rho_{3}(x)$. It can be seen that $\rho_{1}$ and $\rho_{2}$ are negligibly different from each other, but significantly different from $\rho_{3}$; but according to ATI, $\rho_{1}$ and $\rho_{2}$ are different while $\rho_{2}$ and $\rho_{3}$ are the same, because $\rho_{1}$ is absolutely translation invariant but $\rho_{2}$ and $\rho_{3}$ are not. This sharply-defined and sometimes incorrect categorisation may result in an unfair evaluation of risk measures. In fact, if a risk measure is absolutely translation invariant, it has good mathematical and financial characteristics; but if it is not, no firm conclusions can be drawn. Such a defect cannot be found in the axiom of STI, because if a risk measure is not sensibly translation invariant, it surely cannot be relied on in financial applications.

\subsection{Sensible positive homogeneity (SPH)}

This axiom tries to address two defects that are related to APH. These defects are:

1. The mathematical stringency that was discussed for ATI can again be seen in absolute positive homogeneity (APH). As an example, for unreasonable results of such rigidity consider two risk measures of variance and standard deviation that are to some extent the same. However,according to APH, standard deviation is absolutely positive homogenous, but variance is not. This instance implies that APH is not very intelligent in handling risk measures with the same logic but with different formulations.

2. There is some kind of paradox between APH and monotonicity. Assume $x>0$ and $m<1$, so $0<m x<x$. In this condition, according to monotonicity $\rho(m x)>\rho(x)$; but according to $\mathrm{APH}, \rho(x)>\rho(m x)$. As an another case, consider the condition in which $x>0$ and $m>1$; then we shall have $0<x<m x$. So according to monotonicity, $\rho(x)>\rho(m x)$; but according to APH, $\rho(m x)>\rho(x)$. After taking into account the discussed paradox, since the axiom of monotonicity is fully based on reason, APH is the axiom that should be revised. The reason for such irrational outcomes from APH is ignorance of the assets returns signals.

To remove the defects of $\mathrm{APH}$, the coefficient of $m$ is replaced by $g(m)$, while on the other hand its formulation is segregated on the basis of the returns signals. So the given risk measure of $\rho$ is 'sensibly positive homogenous' if $\rho(m x)=g(m) \rho(x)$, where $g(m)$ is as specified by Eq.2. if $x \geq 0$ and by Eq. 3 if $x \leq 0$.

$$
\begin{aligned}
& \left\{\begin{array}{rl}
0<g(m)<1 & \text { if } m>1 \\
g(m)>1 & \text { if } m<1
\end{array} ; \text { and } \frac{\partial g(m)}{\partial m}<0,\right. \\
& \left\{\begin{array}{rl}
g(m)>1 & \text { if } m>1 \\
0<g(m)<1 & \text { if } m<1
\end{array} ; \text { and } \frac{\partial g(m)}{\partial m}>0\right.
\end{aligned}
$$


Whereas for the two versions of coherency, the axioms of subadditivity and monotonicity are exactly the same, and ATI is a special case of STI, the fourth axiom represents the major difference between absolute and sensible coherency.

\section{LPM and sensible coherency}

This section is intended to produce a case in which, for a particular risk measure, the state of being sensibly coherent is investigated, and on the other hand to present a new set of proofs on the competence of LPMs. Part 4.1 introduces the family of LPMs, and parts 4.2 to 4.5 explore whether LPM of the first order has absolute, sensible, or no shape of coherency.

\subsection{LPM}

Generally symmetrical risk measures like variance can be clearly dismissed in favour of shortfall measures like LPMs [17]. For early research on the topic of shortfall measures, Roy [18], Markowitz [19], and Mao [20] can be named. One class of shortfall measures of risk that is consistent with the definition of increasing risk for arbitrary probability distributions is LPM. The appeal of these risk measures has been based, in part, on their consistency with the way individuals actually perceive risk [21], and so the LPM approach is of special importance for applications to financial decision-making [17]. Seminal references for the concept of LPM are Bawa [22,23], Fishburn [24], and Bawa and Lindenberg [25], while more recent papers are Harlow and Rao [21], and Grootveld and Hallerbach [26].

This category of risk measures is significantly efficient, both theoretically and practically. Bawa $[22,23]$ has shown that for every scalar $\tau$ and for every return distribution belonging to a certain class of distributions, the Mean-LPM model will produce portfolios that will dominate all other portfolios according to the concept of stochastic dominance. Harlow et al. [27] recommend application of LPM for the development of asset pricing models, and believe that the generalised Mean-LPM model overcomes the limited appeal of earlier formulations - and moreover, it is found that for a large set of target rates, their generalised Mean-LPM framework cannot be rejected in favour of an unspecified alternative. Unser [17] also states that empirical and simulation studies show the superiority of Mean-LPM based portfolio selection criteria in the traditional mean-variance based approach.

The LPM of order $\alpha$ around $\tau$ is defined by Eq.4.

$$
L P M_{\alpha}(\tau ; R)=\int_{-\infty}^{\tau}(\tau-R)^{\alpha} d F(R)=E\left\{(\max [\tau-R, 0])^{\alpha}\right\}
$$

where $F(R)$ is the cumulative distribution function of the investment return $R ; \tau$ is the target parameter; and $\alpha$ determines the weight the investor places on deviations. An interesting advantage of LPM is its totality in comparison with the other risk measures, in the way that Harlow et al. [27] state that a large class of extant pricing models using risk measures like variance, semivariance, semideviation, probability of loss, etc. become special cases of the Mean-LPM framework. For example, if $\tau$ equals the risk-free interest rate, $R_{f}$, the Bawa-Lindenberg model is obtained directly; and if in addition to $\tau=R_{f}$, returns have normal distribution and $\alpha=2$, the Sharpe-Lintner-Mossin CAPM model can be achieved [21]. The semi-variance corresponds to the LPM with $\tau=E(R)$ and $\alpha=2$; the probability of loss that corresponds to Roy's safety, and the expected loss, are achieved from LPM if $\alpha=0$ and $\alpha=1$ respectively.

It is interesting to note that LPM - with all of the above-mentioned advantages - does not have absolute coherency, because it violates the axioms of ATI and APH. This point again emphasises the fact that sometimes the concept of absolute coherency is not fair in evaluating risk measures, and needs some kind of revision - as is done in this paper. 
Fishburn [24] has shown that for LPM, if $\alpha=1$, it suits a risk-neutral investor, i.e. $\alpha=1$ separates risk-seeking $(0<\alpha<1)$ from risk averse behaviour $(\alpha>1)$. Building on this point, LPM of $\alpha=1$, with no constraint on $\tau$ (as shown in Eq.5) is chosen for further discussion.

$$
L P M_{1}(\tau ; R)=\int_{-\infty}^{\tau}(\tau-R) d F(R)=E\{\max (\tau-R, 0)\} .
$$

\subsection{The LPM and Subadditivity}

$\operatorname{LPM}_{1}(\tau, R)$ is subadditive if the inequality $(6)$ is maintained.

$$
\int_{-\infty}^{R_{\text {nec }}}\left(R_{\text {nec }}-(x+y)\right) f_{X+Y}(x+y) d(x+y) \leq \int_{-\infty}^{R_{\text {nec }}}\left(R_{\text {nec }}-x\right) f_{X}(x) d x+\int_{-\infty}^{R_{\text {nec }}}\left(R_{\text {nec }}-y\right) f_{Y}(y) d y .
$$

The inequality (6) can be rewritten as the inequality (7).

$$
E\left(\operatorname{Max}\left(R_{\text {nec }}-(x+y), 0\right)\right) \leq E\left(\operatorname{Max}\left(R_{\text {nec }}-x, 0\right)\right)+E\left(\operatorname{Max}\left(R_{\text {nec }}-y, 0\right)\right) \cdot
$$

Since for every convex function of $f$ we have the inequality (8), [28]

$$
f(\alpha x+(1-\alpha) y) \leq \alpha f(x)+(1-\alpha) f(y),
$$

and whereas $E(\operatorname{Max}(u, 0))$ is also a convex function, inequality (9) can be derived.

$$
E\left(\operatorname{Max}\left(\alpha\left(R_{\text {nec }}-x\right)+(1-\alpha)\left(R_{\text {nec }}-y\right), 0\right)\right) \leq \alpha E\left(\operatorname{Max}\left(\left(R_{\text {nec }}-x\right), 0\right)\right)+(1-\alpha) E\left(\operatorname{Max}\left(\left(R_{\text {nec }}-y\right), 0\right)\right)
$$

In the last inequality, if $\alpha=\frac{1}{2}$ the inequality (10) is achieved as follows:

$$
E\left(\operatorname{Max}\left(R_{\text {nec }}-\frac{x+y}{2}, 0\right)\right) \leq \frac{1}{2} E\left(\operatorname{Max}\left(\left(R_{\text {nec }}-x\right), 0\right)\right)+\frac{1}{2} E\left(\operatorname{Max}\left(\left(R_{\text {nec }}-y\right), 0\right)\right)
$$

After multiplying the above inequality by 2 , inequality (11) results.

$$
E\left(\operatorname{Max}\left(2 R_{\text {nec }}-(x+y), 0\right)\right) \leq E\left(\operatorname{Max}\left(\left(R_{\text {nec }}-x\right), 0\right)\right)+E\left(\operatorname{Max}\left(\left(R_{\text {nec }}-y\right), 0\right)\right)
$$

Since the inequality (12) is obvious, the inequality (11) alongside (12) assures subadditivity of the discussed risk measure.

$$
E\left(\max \left(2 R_{\text {nec }}-(x+y), 0\right)\right) \geq E\left(\max \left(R_{\text {nec }}-(x+y), 0\right)\right)
$$

\subsection{The LPM and Monotonicity}

$L P M_{1}(\tau, R)$ is monotonous if for $x>y$ the inequality (13) can be concluded.

$$
\int_{-\infty}^{R_{n e c}}\left(R_{n e c}-x\right) f_{X}(x) d x \leq \int_{-\infty}^{R_{n e c}}\left(R_{n e c}-y\right) f_{Y}(y) d y
$$

As a first step, the inequality (13) is rewritten as the inequality (14).

$$
E\left(\operatorname{Max}\left(R_{\text {nec }}-x, 0\right)\right) \leq E\left(\operatorname{Max}\left(R_{\text {nec }}-y, 0\right)\right) .
$$

Lemma. If $x \leq y$ then $E(x) \leq E(y)$ 
Proof.

Obviously $\operatorname{Min}(x) \leq E(x) \leq \operatorname{Max}(x)$ and $\operatorname{Min}(y) \leq E(y) \leq \operatorname{Max}(y)$. On the other hand, for the variables of $x$ and $y, \operatorname{Max}(x) \leq \operatorname{Min}(y)$. Since $E(x) \leq \operatorname{Max}(x)$ and $\operatorname{Min}(y) \leq E(y)$, it is concluded that $E(x) \leq E(y)$.

Since $x \geq y$ then $R_{\text {nec }}-x \leq R_{\text {nec }}-y$, and consequently $\operatorname{Max}\left(R_{\text {nec }}-x, 0\right) \leq \operatorname{Max}\left(R_{\text {nec }}-y, 0\right)$. Based on the Lemma, it can be concluded that $E\left(\operatorname{Max}\left(R_{\text {nec }}-x, 0\right)\right) \leq E\left(\operatorname{Max}\left(R_{\text {nec }}-y, 0\right)\right)$. So $\operatorname{LPM}_{1}(\tau, R)$ is also a monotonous risk measure.

\subsection{The LPM and STI}

To determine whether $\operatorname{LPM}_{1}(\tau, R)$ is absolutely or sensibly translated invariance, or neither of them, $\rho(x+a)$ should be calculated as is done in Eq. 15 .

$$
\begin{aligned}
\rho(x+a) & =\int_{-\infty}^{R_{n e c}-a}\left(R_{\text {nec }}-(x+a)\right) f_{X}(x) d x=\int_{-\infty}^{R_{\text {nec }}-a}\left(R_{\text {nec }}-x\right) f_{X}(x) d x-a \int_{-\infty}^{R_{n e c}-a} f_{X}(x) d x . \\
& =\int_{-\infty}^{R_{n e c}}\left(R_{\text {nec }}-x\right) f_{X}(x) d x-\int_{R_{n e c}-a}^{R_{n e c}}\left(R_{\text {nec }}-x\right) f_{X}(x) d x-a \int_{-\infty}^{R_{n e c}-a} f_{X}(x) d x \\
& =\rho(x)-\left[\int_{R_{n e c}-a}^{R_{n e c}}\left(R_{\text {nec }}-x\right) f_{X}(x) d x+a \int_{-\infty}^{R_{n e c}-a} f_{X}(x) d x\right]
\end{aligned}
$$

As can be seen in Eq.15, $g(a)=\int_{R_{\text {nec }}-a}^{R_{n e c}}\left(R_{\text {nec }}-x\right) f_{X}(x) d x+a \int_{-\infty}^{R_{n e c}-a} f_{X}(x) d x$ so because $g(a) \neq a, L P M_{1}(\tau, R)$ is not absolutely translated invariance but sensibly translated invariance, its $g(a)$ should meet two characteristics. It obviously meets the first one because, if $a>0, g(a)>0$, and if $a<0, g(a)<0$. For the second characteristic, it should be checked whether $\frac{\partial g(a)}{\partial a}$ is positive or negative. Since $\frac{\partial g(a)}{\partial a}=\int_{-\infty}^{R_{\text {mac }}-a} f_{X}(x) d x>0$ it is proved that $L P M_{1}(\tau, R)$ is sensibly translated invariance.

\subsection{The LPM and SPH}

It is apparent that $L P M_{1}(\tau, R)$ does not have $\mathrm{APH}$; but to determine whether or not it has $\mathrm{SPH}$, at the first step $g(m)$ should be calculated. Since there are two methods for presenting $L P M_{1}(\tau, R)$, the $g(m)$ can be formulated both statistically and mathematically. The statistical and mathematical expression of $g(m)=\frac{\rho(m x)}{\rho(x)}$ is shown by Eqs (16) and (17) respectively.

$$
\frac{E\left(\max \left(R_{n e c}-m x, 0\right)\right)}{E\left(\max \left(R_{n e c}-x, 0\right)\right)},
$$

$$
\frac{\int_{-\infty}^{\frac{R_{n e c}}{m}}\left(R_{\text {nec }}-m x\right) f_{X}(x) d x}{\int_{-\infty}^{R_{\text {nec }}}\left(R_{\text {nec }}-x\right) f_{X}(x) d x} .
$$

If $x \geq 0$ and $m>1$ or $x \leq 0$ and $m<1$, for $\operatorname{LPM}_{1}(\tau, R)$, on the basis of what follows and Eq. 16 it is concluded that $0<g(m)<1$. 


$$
\begin{aligned}
& x<m x \Rightarrow R_{\text {nec }}-x>R_{\text {nec }}-m x \Rightarrow \operatorname{Max}\left(R_{\text {nec }}-x, 0\right)>\operatorname{Max}\left(R_{\text {nec }}-m x, 0\right) \Rightarrow \\
& E\left(\operatorname{Max}\left(R_{\text {nec }}-x, 0\right)\right)>E\left(\operatorname{Max}\left(R_{\text {nec }}-m x, 0\right)\right) .
\end{aligned}
$$

And if $x \geq 0$ and $m<1$ or $x \leq 0$ and $m>1$, for $\operatorname{LPM}_{1}(\tau, R)$, on the basis of what follows and Eq. 16 it is concluded that $g(m)>1$.

$$
\begin{aligned}
& m x<x \Rightarrow R_{\text {nec }}-m x>R_{\text {nec }}-x \Rightarrow \operatorname{Max}\left(R_{\text {nec }}-m x, 0\right)>\operatorname{Max}\left(R_{\text {nec }}-x, 0\right) \Rightarrow \\
& E\left(\operatorname{Max}\left(R_{\text {nec }}-m x, 0\right)\right)>E\left(\operatorname{Max}\left(R_{\text {nec }}-x, 0\right)\right)
\end{aligned}
$$

The other thing that should be checked is the signal of $\frac{\partial g(m)}{\partial m}$ that is different for $x \geq 0$ and $x \leq 0$. To check this point, the mathematical definition of $g(m)$ (Eq.17) will be used. The first derivative of $g(m)$ is shown by Eq. 18 .

$$
\frac{\partial g(m)}{\partial m}=\frac{-\int_{-\infty}^{\frac{R_{m e c}}{m}} x f_{X}(x) d x}{\int_{-\infty}^{R_{\text {nec }}}\left(R_{\text {nec }}-x\right) f_{X}(x) d x}
$$

According to the above equation, for $x \geq 0$, we have $\frac{\partial g(m)}{\partial m}<0$ and for $x \leq 0$, we have $\frac{\partial g(m)}{\partial m}>0$. So it is concluded that LPM of the first order is sensibly positively homogenous.

\section{CONCLUSION}

Beside general reviews of the field of portfolio selection, concepts of coherency, and the risk measure of LPM, the main contribution of this paper is to present a new concept for evaluating risk measures entitled 'sensible coherency'. To be honest, the new concept is a new version of the popular concept of 'coherency'. The new concept is exactly the same as the old one on the axioms of subadditivity and monotonicity; to some extent it is different on the axiom of translation invariance; and it is completely different on the fourth axiom. The changes are made to make the concept more practical and give it greater potential to yield good results. Lastly, it has been checked that, whether LPM of the first order is sensibly coherent or not, it was proved to be sensibly coherent but not absolutely coherent.

\section{REFERENCES}

[1] Markowitz, H.M. 1952. Portfolio selection. Journal of Finance, 25, 71-79.

[2] Konno, H. \& Suzuki, K. 1992. A fast algorithm for solving large scale mean-variance models by compact factorization of covariance matrices. Journal of the Operations Research Society of Japan, 35, 93-104.

[3] Perold, A.F. 1984. Large-scale portfolio optimization. Journal of Management Science, 30, 1143-1160.

[4] Artzner, P., Delbaen,F., Eber, J.M. \& Heath, D. 1997. Thinking coherently. Journal of Risk, 10, 68-71.

[5] Artzner, P., Delbaen,F., Eber, J.M. \& Heath, D. 19971999. Coherent measures of risk. Journal of Mathematical Finance, 9, 203-228.

[6] Bertsimas, D., Lauprete, G.J. \& Samarov, A. 2000. Shortfall as a risk measure: Properties, optimization and applications. Working paper, Sloan School of Management, MIT, Cambridge.

[7] Delbaen, F. 2000. Coherent risk measures on general probability spaces. In: Sandmann, K. and Schonbucher, P.J. (eds), Advances in finance and stochastics. Essays in honour of Dieter Sondermann. Springer-Verlag, Berlin, pp. 1-37. 
[8] Kusuoka, S. 2001. On law invariant coherent risk measures. Journal of Advances in Mathematical Economics, 3, 83-95.

[9] Acerbi, C. 2002. Spectral measures of risk: A coherent representation of subjective risk aversion. Journal of Banking and Finance, 26, 1505-1518.

[10] Fritelli, M. \& Rosazza Gianin, E. 2002. Putting order in risk measures. Journal of Banking and Finance, 26, 1473-1486.

[11] Acerbi, C. \& Tasche, D. 2002. On the coherence of expected shortfall. Journal of Banking and Finance, 26, 1487-1503.

[12] Szego, G. 2002. Measures of risk. Journal of Banking and Finance, 26, 1253-1272.

[13] Inoue, A. 2003. On the worst conditional expectation. Journal of Mathematical Analysis and Applications, 286, 237-247.

[14] Pelessoni, R. \& Vicig, P. 2001. Coherent risk measures and upper previsions. In: Second International Symposium on Imprecise Probabilities and Their Applications, Ithaca, New York.

[15] Giannopoulos, K. \& Tunaru, R. 2005. Coherent risk measures under filtered historical simulation. Journal of Banking and Finance, 29, 979-996.

[16] Embrechts, P., McNeil, A. \& Straumann, D. 2002. Correlation and dependence in risk management: Properties and pitfalls. In: Dempster, M.A.H. (ed.), Risk management: Value at risk and beyond. Cambridge University Press, Cambridge, pp. 176-223.

[17] Unser, M. 2000. Lower partial moments as measures of perceived risk: An experimental study. Journal of Economic Psychology, 21, 253-280.

[18] Roy, A. 1952. Safety-first and the holding of assets. Journal of Econometrica, 20, 431-449.

[19] Markowitz, H.M. 1959. Portfolio selection: Efficient diversification of investments. Wiley, New York.

[20] Mao, J. 1970. Survey of capital budgeting theory and practice. Journal of Finance, 25, 349-360.

[21] Harlow, W.V. \& Rao, R.K.S. 1999. Asset pricing in a generalized mean-lower partial moment framework: Theory and evidence. The Journal of Financial and Quantitative Analysis, 24, 285-311.

[22] Bawa, V.S. 1975. Optimal rules for ordering uncertain prospects. Journal of Financial Economics, 2, 95-121.

[23] Bawa, V.S. 1978. Safety-first, stochastic dominance, and optimal portfolio choice. Journal of Financial and Quantitative Analysis, 13, 255-271.

[24] Fishburn, P.C. 1977. Mean-risk analysis with risk associated with below-target returns. Journal of The American Economic Review, 116-126.

[25] Bawa, V.S. \& Lindenberg, E.B. 1977. Capital market equilibrium in a mean-lower partial moment framework. Journal of Financial Economics, 5, 189-200.

[26] Grootveld, H. \& Hallerbach, W. 1999. Variance vs. downside risk: Is there really that much difference? European Journal of Operations Research, 114, 304-319.

[27] Boyd, S. \& Vandenberghe, L. 2004. Convex optimization. Cambridge University Press, New York. 
http://sajie.journals.ac.za 